\title{
Bonding Properties of Isolated Metal Atoms on Two-Dimensional Oxides
}

Sergio Tosoni and Gianfranco Pacchioni

Dipartimento di Scienza dei Materiali, Università di Milano-Bicocca, via R. Cozzi 55, 20125 Milano, Italy

\section{Supporting Information}

\section{Computational Details}

Table S1. List of PAW pseudopotentials adopted in each case in the present work

\begin{tabular}{|c|c|c|c|c|}
\hline & Symbol & PAW potential & $\mathrm{N}$ electrons & Electronic configuration \\
\hline \multirow[t]{3}{*}{ Adatoms } & $\mathrm{K}$ & PAW_PBE K_sv & 9 & $3 s^{2} 3 p^{6} 4 s^{1}$ \\
\hline & $\mathrm{Pt}$ & PAW_PBE Pt & 10 & $5 d^{9} 6 s^{1}$ \\
\hline & $\mathrm{Au}$ & PAW_PBE $\mathrm{Au}$ & 11 & $5 d^{10} 6 s^{1}$ \\
\hline \multirow[t]{4}{*}{$\mathrm{MgO} /(\mathrm{Ag}, \mathrm{Mo})$} & $\mathrm{O}$ & PAW_PBE O & 6 & $2 s^{2} 2 p^{4}$ \\
\hline & $\mathrm{Mg}$ & PAW_PBE Mg_pv & 8 & $2 p^{6} 3 s^{2}$ \\
\hline & $\mathrm{Ag}$ & PAW_PBE Ag & 11 & $4 d^{10} 5 s^{1}$ \\
\hline & Mo & PAW_PBE Mo_pv & 12 & $4 p^{6} 4 d^{5} 5 s^{1}$ \\
\hline \multirow[t]{5}{*}{$\mathrm{ZnO} /(\mathrm{Cu}, \mathrm{Ag}, \mathrm{Au})$} & $\mathrm{O}$ & PAW_PBE O & 6 & $2 s^{2} 2 p^{4}$ \\
\hline & $\mathrm{Zn}$ & PAW_PBE Zn & 12 & $3 d^{10} 4 s^{2}$ \\
\hline & $\mathrm{Cu}$ & PAW_PBE Cu & 11 & $3 d^{10} 4 s^{1}$ \\
\hline & $\mathrm{Ag}$ & PAW_PBE Ag_pv & 17 & $4 p^{6} 4 d^{10} 5 s^{1}$ \\
\hline & $\mathrm{Au}$ & PAW_PBE Au & 11 & $5 d^{10} 6 s^{1}$ \\
\hline \multirow[t]{4}{*}{$\mathrm{SiO}_{2} /(\mathrm{Pt}, \mathrm{Ru})$} & $\mathrm{O}$ & PAW_PBE O & 6 & $2 s^{2} 2 p^{4}$ \\
\hline & $\mathrm{Si}$ & PAW_PBE Si & 4 & $3 s^{2} 3 p^{2}$ \\
\hline & $\mathrm{Pt}$ & PAW_PBE Pt & 10 & $5 d^{9} 6 s^{1}$ \\
\hline & $\mathrm{Ru}$ & PAW_PBE Ru & 8 & $4 d^{7} 5 s^{1}$ \\
\hline \multirow[t]{4}{*}{$\mathrm{TiO}_{2} /(\mathrm{Pt}, \mathrm{Ag})$} & $\mathrm{O}$ & PAW_PBE O & 6 & $2 s^{2} 2 p^{4}$ \\
\hline & $\mathrm{Ti}$ & PAW_PBE Ti_pv & 10 & $3 p^{6} 3 d^{3} 4 s^{1}$ \\
\hline & $\mathrm{Pt}$ & PAW_PBE Pt & 10 & $5 d^{9} 6 s^{1}$ \\
\hline & $\mathrm{Ag}$ & PAW_PBEAg_pv & 17 & $4 p^{6} 4 d^{10} 5 s^{1}$ \\
\hline \multirow[t]{3}{*}{$\mathrm{ZrO}_{2} /(\mathrm{Pt}, \mathrm{ZrPt})$} & $\mathrm{O}$ & PAW_PBE O & 6 & $2 s^{2} 2 p^{4}$ \\
\hline & $\mathrm{Zr}$ & PAW_PBE Zr_sv & 12 & $4 s^{2} 4 p^{6} 4 d^{2} 5 s^{2}$ \\
\hline & $\mathrm{Pt}$ & PAW_PBE Pt & 10 & $5 d^{9} 6 s^{1}$ \\
\hline
\end{tabular}


Table S2. Adopted supercell and mesh of $\mathrm{K}$ points, residual strain released on the oxide film, minimum adatom-adatom lateral distance $\left(\mathrm{R}_{\min }, \AA\right)$.

\begin{tabular}{|c|c|c|c|c|}
\hline & Adopted supercell & K points & Strain $(\%)^{\mathrm{a}}$ & $\mathrm{R}_{\min }(\AA)$ \\
\hline $\mathrm{MgO}$ free-standing & $5 \times 5 \mathrm{MgO}(100)$ & $\Gamma$ only & $0 \%$ & 14.97 \\
\hline $\mathrm{MgO} / \mathrm{Ag}$ & $4 \mathrm{x} 4 \mathrm{MgO}(100) / 4 \mathrm{x} 4 \mathrm{Ag}(100)$ & $3 \times 3 \times 1$ & $-1.7 \% \mathrm{C}$ & 11.77 \\
\hline $\mathrm{MgO} / \mathrm{Mo}$ & $4 \times 4 \mathrm{MgO}(100) / 4 x 4 \mathrm{Mo}(100)$ & $3 \times 3 \times 1$ & $+5.6 \% \mathrm{~T}$ & 12.65 \\
\hline $\mathrm{ZnO}$ free-standing & $3 \times 3 \mathrm{ZnO}$ graphitic bilayer & $\Gamma$ only & $0 \%$ & 9.84 \\
\hline \multirow[t]{2}{*}{$\mathrm{ZnO} / \mathrm{Cu}$} & $3 \times 3 \mathrm{ZnO} / 4 \times 4 \mathrm{Cu}(111)$ & $4 \times 4 \times 1$ & $+2.3 \% \mathrm{~T}$ & 10.10 \\
\hline & $6 \times 6 \mathrm{ZnO} / 8 \mathrm{x} 8 \mathrm{Cu}(111)$ & $\Gamma$ only & & 20.20 \\
\hline $\mathrm{ZnO} / \mathrm{Ag}$ & 7x7 ZnO/ 8x8 Ag(111) & $\Gamma$ only & $+1.9 \% \mathrm{~T}$ & 23.46 \\
\hline $\mathrm{ZnO} / \mathrm{Au}$ & $7 \times 7 \mathrm{ZnO} / 8 \times 8 \mathrm{Ag}(111)$ & $\Gamma$ only & $+1.2 \% \mathrm{~T}$ & 23.29 \\
\hline $\mathrm{SiO}_{2}$ free-standing & $2 \times 2 \mathrm{SiO}_{2}$ bilayer & $\Gamma$ only & $0 \%$ & 10.62 \\
\hline \multirow[t]{2}{*}{$\mathrm{SiO}_{2} / \mathrm{Pt}$} & $2 \times 2 \mathrm{SiO}_{2} / 4 \times 4 \mathrm{Pt}(111)$ & $3 \times 3 \times 1$ & $+4.6 \% \mathrm{~T}$ & 11.1 \\
\hline & $4 \times 4 \mathrm{SiO}_{2} / 8 \times 8 \mathrm{Pt}(111)$ & $\Gamma$ only & & 22.3 \\
\hline \multirow[t]{2}{*}{$\mathrm{SiO}_{2} / \mathrm{Ru}$} & $2 \times 2 \mathrm{SiO}_{2} / 4 \times 4 \mathrm{Ru}(0001)$ & $3 \times 3 \times 1$ & $+2.8 \% \mathrm{~T}$ & 10.9 \\
\hline & $4 \times 4 \mathrm{SiO}_{2} / 8 \times 8 \mathrm{Ru}(0001)$ & $\Gamma$ only & & 22.0 \\
\hline $\mathrm{TiO}_{2}$ free-standing & $3 \times 4 \mathrm{TiO}_{2}$ lepidocrocite & $\Gamma$ only & $0 \%$ & 11.40 \\
\hline $\mathrm{TiO}_{2} / \mathrm{Pt}$ & $\begin{array}{l}4 \times 4 \quad \mathrm{TiO}_{2} / \sqrt{7} \times \sqrt{7} \quad \mathrm{R} \quad 19.1^{\circ} \\
\mathrm{Pt}(111)\end{array}$ & $4 \times 4 \times 1$ & $\begin{array}{l}-3.1 \% \quad \mathrm{C} \\
+4.8 \% \mathrm{~T}\end{array}$ & 14.72 \\
\hline $\mathrm{TiO}_{2} / \mathrm{Ag}$ & $4 \times 4 \mathrm{TiO}_{2} / 5 \times 4 \mathrm{Ag}(100)$ & $2 \times 2 \times 1$ & $\begin{array}{l}-0.1 \% \quad \mathrm{C} \\
+6.2 \% \mathrm{~T}\end{array}$ & 11.73 \\
\hline $\mathrm{ZrO}_{2}$ free-standing & $5 \times 5 \mathrm{ZrO}_{2} \mathrm{ML}$ & $\Gamma$ only & $0 \%$ & 16.76 \\
\hline $\mathrm{ZrO}_{2} / \mathrm{Pt}$ & $4 \times 4 \mathrm{ZrO}_{2} / 5 \times 5 \mathrm{Pt}(111)$ & $2 \times 2 \times 1$ & $+3.5 \% \mathrm{~T}$ & 13.89 \\
\hline $\mathrm{ZrO}_{2} / \mathrm{ZrPt}$ & $5 \times 5 \mathrm{ZrO}_{2} / 3 \times 3 \mathrm{ZrPt}(0001)$ & $2 \times 2 \times 1$ & $+1.4 \% \mathrm{~T}$ & 17.00 \\
\hline
\end{tabular}

${ }^{\mathrm{a}} \mathrm{C}$ and $\mathrm{T}$ stand for Compressive or Tensile strain.

\section{Comprehensive list of all local minima}

Table S3. Adsorption of K, Pt and Au single atoms on free-standing MgO(100). Adsorption energy (De, eV), total magnetization $(\mathrm{M},|\mathrm{e}|)$, Bader charge of the adsorbed atom $\left(\mathrm{q}_{\mathrm{A}},|\mathrm{e}|\right)$, adatom-oxygen minimum distance $\left(\mathrm{R}_{\mathrm{O}}, \AA\right)$ and adatom-Mg minimum distance $\left(\mathrm{R}_{\mathrm{Mg}}, \AA\right)$.

\begin{tabular}{llllllll}
\hline Support & Atom & Site & $\mathrm{De}(\mathrm{eV})$ & $\mathrm{M}(|\mathrm{e}|)$ & $\mathrm{q}_{\mathrm{A}}(|\mathrm{e}|)$ & $\mathrm{R}_{\mathrm{O}}(\AA)$ & $\mathrm{R}_{\mathrm{Mg}}(\AA)$ \\
\hline $\mathrm{MgO}(100)$ & $\mathrm{K}$ & $\mathrm{O}$ & -0.28 & 1.00 & +0.30 & 2.88 & 3.71 \\
& & Hollow & -0.21 & 1.00 & +0.30 & 3.30 & 3.44 \\
& $\mathrm{Pt}$ & $\mathrm{O}$ & -2.55 & 0.04 & -0.35 & 1.97 & 2.92 \\
& $\mathrm{Mg}$ & -0.69 & 1.92 & -0.24 & 3.36 & 2.62 \\
& & $\mathrm{Hollow}$ & -1.57 & 2.00 & -0.38 & 2.45 & 2.59 \\
& $\mathrm{Au}$ & $\mathrm{O}$ & -1.09 & 1.00 & -0.29 & 2.26 & 3.13 \\
\hline
\end{tabular}


Table S4. Adsorption of K, Pt and Au single atoms on MgO BL and ML supported on $\mathrm{Ag}(100)$. Adsorption energy $(\mathrm{De}, \mathrm{eV})$, total magnetization $(\mathrm{M},|\mathrm{e}|)$, Bader charge of the adsorbed atom $\left(\mathrm{q}_{\mathrm{A}},|\mathrm{e}|\right)$, adatom-oxygen minimum distance $\left(\mathrm{R}_{\mathrm{O}}, \AA\right)$ and adatom-Mg minimum distance $\left(\mathrm{R}_{\mathrm{Mg}}, \AA\right)$.

\begin{tabular}{|c|c|c|c|c|c|c|c|}
\hline Support & Atom & Site & De $(e V)$ & $\mathrm{M}(|\mathrm{e}|)$ & $\mathrm{q}_{\mathrm{A}}(|\mathrm{e}|)$ & $\mathrm{R}_{\mathrm{O}}(\AA)$ & $\mathrm{R}_{\mathrm{Mg}}(\AA)$ \\
\hline \multirow[t]{8}{*}{$\mathrm{MgO} \mathrm{ML} / \mathrm{Ag}$} & \multirow[t]{2}{*}{ K } & $\mathrm{O}$ & -1.13 & 0.00 & +0.81 & 2.48 & 3.56 \\
\hline & & Hollow & -1.26 & 0.00 & +0.82 & 2.67 & 3.16 \\
\hline & \multirow[t]{3}{*}{$\mathrm{Pt}$} & $\mathrm{O}$ & -2.85 & 0.00 & -0.69 & 2.10 & 2.82 \\
\hline & & $\mathrm{Mg}$ & -1.85 & 0.73 & -0.83 & 3.54 & 2.53 \\
\hline & & Hollow & -2.22 & 0.54 & -1.00 & 3.07 & 2.63 \\
\hline & \multirow[t]{3}{*}{$\mathrm{Au}$} & $\mathrm{O}$ & -1.99 & 0.00 & -0.77 & 2.76 & 3.07 \\
\hline & & $\mathrm{Mg}$ & -1.81 & 0.00 & -0.74 & 3.56 & 2.59 \\
\hline & & Hollow & -1.99 & 0.00 & -0.78 & 3.16 & 2.78 \\
\hline \multirow[t]{8}{*}{$\mathrm{MgO} \mathrm{BL} / \mathrm{Ag}$} & \multirow[t]{2}{*}{$\mathrm{K}$} & $\mathrm{O}$ & -0.60 & 0.00 & +0.72 & 2.50 & 3.58 \\
\hline & & Hollow & -0.66 & 0.00 & +0.82 & 2.69 & 3.10 \\
\hline & \multirow[t]{3}{*}{$\mathrm{Pt}$} & $\mathrm{O}$ & -2.58 & 0.49 & -0.66 & 2.07 & 2.86 \\
\hline & & $\mathrm{Mg}$ & -1.70 & 0.94 & -0.83 & 3.67 & 2.57 \\
\hline & & Hollow & -1.88 & 0.79 & -0.96 & 3.18 & 2.70 \\
\hline & \multirow[t]{3}{*}{$\mathrm{Au}$} & $\mathrm{O}$ & -1.70 & 0.00 & -0.82 & 2.80 & 3.17 \\
\hline & & $\mathrm{Mg}$ & -1.74 & 0.00 & -0.81 & 3.65 & 2.59 \\
\hline & & Hollow & -1.82 & 0.00 & -0.84 & 3.20 & 2.83 \\
\hline
\end{tabular}


Table S5. Adsorption of K, Pt and Au single atoms on MgO BL and ML supported on Mo(100). Adsorption energy $(\mathrm{De}, \mathrm{eV})$, total magnetization $(\mathrm{M},|\mathrm{e}|)$, Bader charge of the adsorbed atom $\left(\mathrm{q}_{\mathrm{A}},|\mathrm{e}|\right)$, adatom-oxygen minimum distance $\left(\mathrm{R}_{\mathrm{O}}, \AA\right)$ and adatom-Mg minimum distance $\left(\mathrm{R}_{\mathrm{Mg}}, \AA\right)$.

\begin{tabular}{|c|c|c|c|c|c|c|c|}
\hline Support & Atom & Site & De $(\mathrm{eV})$ & $\mathrm{M}(|\mathrm{e}|)$ & $\mathrm{q}_{\mathrm{A}}(|\mathrm{e}|)$ & $\mathrm{R}_{\mathrm{O}}(\AA)$ & $\mathrm{R}_{\mathrm{Mg}}(\AA)$ \\
\hline \multirow[t]{8}{*}{$\mathrm{MgO} \mathrm{ML} / \mathrm{Mo}$} & \multirow[t]{2}{*}{$\mathrm{K}$} & $\mathrm{O}$ & -0.50 & 0.00 & +0.47 & 2.64 & 3.72 \\
\hline & & Hollow & -0.45 & 0.01 & +0.63 & 2.89 & 3.27 \\
\hline & \multirow[t]{3}{*}{$\mathrm{Pt}$} & $\mathrm{O}$ & -3.57 & 0.38 & -1.26 & 2.42 & 2.92 \\
\hline & & $\mathrm{Mg}$ & -3.23 & 0.37 & -1.11 & 3.75 & 2.40 \\
\hline & & Hollow & -4.09 & 0.08 & -1.31 & 3.14 & 2.55 \\
\hline & \multirow[t]{3}{*}{$\mathrm{Au}$} & $\mathrm{O}$ & -2.41 & 0.06 & -0.79 & 2.64 & 3.27 \\
\hline & & $\mathrm{Mg}$ & -2.89 & 0.00 & -0.79 & 3.74 & 2.52 \\
\hline & & Hollow & -3.17 & 0.00 & -0.81 & 3.10 & 2.75 \\
\hline \multirow[t]{8}{*}{ MgO BL/Mo } & \multirow[t]{2}{*}{ K } & $\mathrm{O}$ & -0.50 & 0.43 & +0.32 & 2.83 & 3.71 \\
\hline & & Hollow & -0.39 & 0.58 & +0.25 & 3.59 & 3.62 \\
\hline & \multirow[t]{3}{*}{$\mathrm{Pt}$} & $\mathrm{O}$ & -3.65 & 0.95 & -0.96 & 2.20 & 2.89 \\
\hline & & $\mathrm{Mg}$ & -2.90 & 0.61 & -1.01 & 3.65 & 2.49 \\
\hline & & Hollow & -3.29 & 0.38 & -1.23 & 3.14 & 2.61 \\
\hline & \multirow[t]{3}{*}{$\mathrm{Au}$} & $\mathrm{O}$ & -2.57 & 0.00 & -0.82 & 2.73 & 3.22 \\
\hline & & $\mathrm{Mg}$ & -2.71 & 0.00 & -0.81 & 3.64 & 2.58 \\
\hline & & Hollow & -2.84 & 0.02 & -0.84 & 3.14 & 2.81 \\
\hline
\end{tabular}


Table S6. Adsorption of $\mathrm{K}, \mathrm{Pt}$ and $\mathrm{Au}$ single atoms on free-standing $\mathrm{ZnO} \mathrm{BL}$ and $\mathrm{ZnO} \mathrm{BL} / \mathrm{M}(111)[\mathrm{M}=\mathrm{Cu}$, $\mathrm{Ag}, \mathrm{Au}]$. Adsorption energy $(\mathrm{De}, \mathrm{eV})$, total magnetization $(\mathrm{M},|\mathrm{e}|)$, Bader charge of the adsorbed atom $\left(\mathrm{q}_{\mathrm{A}},|\mathrm{e}|\right)$, adatom-oxygen minimum distance $\left(\mathrm{R}_{\mathrm{O}}, \AA\right)$ and adatom- $\mathrm{Zn}$ minimum distance $\left(\mathrm{R}_{\mathrm{Zn}}, \AA\right)$.

\begin{tabular}{llllllll}
\hline Support & Atom & Site & $\mathrm{De}(\mathrm{eV})$ & $\mathrm{M}(|\mathrm{e}|)$ & $\mathrm{q}_{\mathrm{A}}(|\mathrm{e}|)$ & $\mathrm{R}_{\mathrm{O}}(\AA)$ & $\mathrm{R}_{\mathrm{Zn}}(\AA)$ \\
\hline $\mathrm{ZnO}-\mathrm{free}$ & $\mathrm{K}$ & Hollow & -1.93 & 0.00 & +0.84 & 2.71 & 3.17 \\
& $\mathrm{Pt}$ & $\mathrm{O}$ & -2.51 & 0.02 & -0.11 & 1.98 & 2.57 \\
& $\mathrm{Au}$ & Bridge & -0.87 & 1.00 & -0.11 & 2.33 & 2.59 \\
$\mathrm{ZnO} / \mathrm{Cu}(111)$ & $\mathrm{K}$ & $\mathrm{Zn}$ & -2.39 & 0.00 & +0.87 & 2.77 & 2.76 \\
& & Hollow & -2.62 & 0.00 & +0.86 & 2.71 & 3.14 \\
& $\mathrm{Pt}$ & $\mathrm{O}$ & -2.69 & 0.48 & -0.42 & 2.31 & 2.53 \\
& $\mathrm{Au}$ & Hollow & -2.10 & 0.00 & -0.46 & 3.16 & 2.67 \\
$\mathrm{ZnO} / \mathrm{Ag}(111)$ & $\mathrm{K}$ & Zn & -2.67 & 0.00 & +0.88 & 2.75 & 2.82 \\
& & Hollow & -2.80 & 0.00 & +0.88 & 2.73 & 3.19 \\
& $\mathrm{Pt}$ & $\mathrm{O}$ & -2.55 & 0.00 & -0.17 & 2.00 & 2.57 \\
& & Hollow & -3.06 & 0.00 & -0.71 & 3.23 & 2.49 \\
& $\mathrm{Au}$ & Hollow & -2.20 & 0.00 & -0.47 & 3.17 & 2.67 \\
$\mathrm{ZnO} / \mathrm{Au}(111)$ & $\mathrm{K}$ & Zn & -3.23 & 0.00 & +0.88 & 2.74 & 2.84 \\
& & Hollow & -3.33 & 0.00 & +0.88 & 2.73 & 3.20 \\
& $\mathrm{Pt}$ & $\mathrm{O}$ & -2.54 & 0.00 & -0.10 & 1.98 & 2.57 \\
& & Hollow & -2.26 & 0.06 & -0.66 & 3.22 & 2.50 \\
& $\mathrm{Au}$ & Hollow & -1.73 & 0.00 & -0.46 & 3.18 & 2.66
\end{tabular}


Table S7. Adsorption of $\mathrm{K}, \mathrm{Pt}$ and $\mathrm{Au}$ single atoms on free-standing $\mathrm{SiO}_{2} \mathrm{BL}, \mathrm{SiO}_{2} / \mathrm{Pt}(111)$ and $\mathrm{SiO}_{2} / \mathrm{Ru}(0001)$. Adsorption energy (De, eV), total magnetization ( $\left.\mathrm{M},|\mathrm{e}|\right)$, Bader charge of the adsorbed atom $\left(\mathrm{q}_{\mathrm{A}},|\mathrm{e}|\right)$, adatom-oxygen minimum distance $\left(\mathrm{R}_{\mathrm{O}}, \AA\right)$ and adatom-Si minimum distance $\left(\mathrm{R}_{\mathrm{Si}}, \AA\right)$.

\begin{tabular}{|c|c|c|c|c|c|c|c|}
\hline Support & Atom & Site & De $(e V)$ & $\mathrm{M}(|\mathrm{e}|)$ & $\mathrm{q}_{\mathrm{A}}(|\mathrm{e}|)$ & $\mathrm{R}_{\mathrm{O}}(\AA)$ & $\mathrm{R}_{\mathrm{Si}}(\AA)$ \\
\hline \multirow[t]{12}{*}{ Free $\mathrm{SiO}_{2}$} & \multirow[t]{4}{*}{$\mathrm{K}$} & O-top & -0.27 & 1.04 & +0.39 & 2.87 & 3.77 \\
\hline & & Si-top & -0.24 & 0.00 & +0.48 & 3.10 & 3.33 \\
\hline & & Bridge & -0.27 & 0.99 & +0.42 & 2.92 & 3.47 \\
\hline & & Hollow & -0.60 & 0.00 & +0.77 & 3.00 & 3.70 \\
\hline & \multirow[t]{4}{*}{$\mathrm{Pt}$} & O-top & -0.50 & 0.09 & -0.04 & 2.03 & 2.97 \\
\hline & & Si-top & -0.15 & 2.00 & +0.01 & 3.13 & 3.45 \\
\hline & & Bridge & -0.26 & 2.00 & -0.00 & 2.51 & 3.36 \\
\hline & & Hollow & -0.19 & 2.00 & +0.03 & 3.46 & 4.14 \\
\hline & \multirow[t]{4}{*}{$\mathrm{Au}$} & O-top & -0.11 & 1.00 & +0.00 & 3.06 & 3.89 \\
\hline & & Si-top & -0.12 & 1.00 & +0.01 & 3.31 & 3.48 \\
\hline & & Bridge & -0.12 & 1.00 & +0.01 & 3.19 & 3.57 \\
\hline & & Hollow & -0.17 & 1.00 & +0.02 & 3.39 & 4.06 \\
\hline \multirow[t]{9}{*}{$\mathrm{SiO}_{2} / \mathrm{Pt}$} & \multirow[t]{4}{*}{ K } & O-top ${ }^{a}$ & -1.12 & 0.31 & +0.71 & 2.61 & 3.56 \\
\hline & & Si-top ${ }^{\mathrm{a}}$ & -1.15 & 0.27 & +0.73 & 2.95 & 3.10 \\
\hline & & Hollow $^{\mathrm{a}}$ & -1.97 & 0.00 & +0.89 & 2.92 & 3.60 \\
\hline & & Hollow ${ }^{\mathrm{b}}$ & -2.81 & 0.12 & +0.89 & 2.96 & 3.65 \\
\hline & $\mathrm{Pt}$ & Bridge & -1.17 & 0.00 & -0.10 & 1.99 & 2.39 \\
\hline & \multirow[t]{4}{*}{$\mathrm{Au}$} & O-top ${ }^{\mathrm{a}}$ & -0.14 & 1.00 & +0.01 & 2.74 & 3.60 \\
\hline & & Si-top ${ }^{a}$ & -0.19 & 1.00 & -0.02 & 3.13 & 3.15 \\
\hline & & Hollow $^{\mathrm{a}}$ & -0.21 & 1.00 & +0.01 & 3.38 & 3.97 \\
\hline & & Hollow ${ }^{\mathrm{b}}$ & -0.12 & 1.00 & +0.01 & 3.39 & 4.01 \\
\hline \multirow[t]{10}{*}{$\mathrm{SiO}_{2} / \mathrm{Ru}$} & \multirow[t]{3}{*}{ K } & Hollow & -1.27 & 0.00 & +0.91 & 2.95 & 3.65 \\
\hline & & Si-top ${ }^{b}$ & -0.64 & 0.00 & +0.73 & 3.03 & 3.22 \\
\hline & & $\mathrm{Ru}(\mathrm{top})^{\mathrm{a}, \mathrm{c}}$ & -2.00 & 0.00 & +0.79 & & \\
\hline & \multirow[t]{4}{*}{$\mathrm{Pt}$} & O-top ${ }^{a}$ & -1.09 & 0.00 & -0.36 & 2.15 & 2.34 \\
\hline & & Si-top ${ }^{a}$ & -1.27 & 1.12 & -0.47 & 2.89 & 2.33 \\
\hline & & Bridge $^{a}$ & -1.25 & 0.79 & -0.50 & 2.42 & 2.32 \\
\hline & & $\mathrm{Ru}(\mathrm{hcp})^{\mathrm{a}, \mathrm{c}}$ & -5.54 & 0.00 & -1.18 & & \\
\hline & \multirow[t]{3}{*}{$\mathrm{Au}$} & Si-top ${ }^{b}$ & -1.25 & 0.00 & -0.61 & 2.95 & 2.41 \\
\hline & & Hollow ${ }^{\mathrm{b}}$ & -0.61 & 0.00 & -0.40 & 3.50 & 4.10 \\
\hline & & $\mathrm{Ru}(\text { top })^{\mathrm{a}, \mathrm{c}}$ & -2.61 & 0.00 & -0.50 & & \\
\hline
\end{tabular}
a) Data obtained on a $2 \times 2$ supercell
c) the adatom is adsorbed at the metal/oxide interface

b) Refinement on a $4 \times 4$ supercell 
Table S8. Adsorption of $\mathrm{K}, \mathrm{Pt}$ and $\mathrm{Au}$ single atoms on free-standing $\mathrm{TiO}_{2}, \mathrm{TiO}_{2} / \mathrm{Pt}(111)$ and $\mathrm{TiO}_{2} / \mathrm{Ag}(100)$. Adsorption energy $(\mathrm{De}, \mathrm{eV})$, total magnetization $(\mathrm{M},|\mathrm{e}|)$, Bader charge of the adsorbed atom $\left(\mathrm{q}_{\mathrm{A}},|\mathrm{e}|\right)$, adatomoxygen minimum distance $\left(\mathrm{R}_{\mathrm{O}}, \AA\right)$ and adatom-Ti minimum distance $\left(\mathrm{R}_{\mathrm{Ti}}, \AA\right)$.

\begin{tabular}{|c|c|c|c|c|c|c|c|}
\hline Support & Atom & Site & $\mathrm{De}(\mathrm{eV})$ & $\mathrm{M}(|\mathrm{e}|)$ & $\mathrm{q}_{\mathrm{A}}(|\mathrm{e}|)$ & $\mathrm{R}_{\mathrm{O}}(\AA)$ & $\mathrm{R}_{\mathrm{Ti}}(\AA)$ \\
\hline \multirow[t]{12}{*}{$\mathrm{TiO}_{2}$ Free } & \multirow[t]{4}{*}{$\bar{K}$} & $\mathrm{O}(2 \mathrm{c})$ & -2.28 & 1.00 & +0.92 & 2.41 & 3.85 \\
\hline & & $\mathrm{O}(4 \mathrm{c})$ & -2.73 & 1.00 & +0.91 & 2.89 & 3.39 \\
\hline & & Ti(up) & -2.56 & 0.58 & +0.92 & 2.63 & 3.33 \\
\hline & & Ti(down) & -2.71 & 1.00 & +0.92 & 2.57 & 3.81 \\
\hline & \multirow[t]{4}{*}{$\mathrm{Pt}$} & $\mathrm{O}(2 \mathrm{c})$ & -1.41 & 0.07 & +0.17 & 1.91 & 3.35 \\
\hline & & $\mathrm{O}(4 \mathrm{c})$ & -1.76 & 0.06 & +0.29 & 2.50 & 2.63 \\
\hline & & Ti(up) & -1.97 & 0.06 & +0.28 & 2.07 & 2.37 \\
\hline & & Ti(down) & -3.21 & 0.07 & +0.08 & 2.05 & 2.79 \\
\hline & \multirow[t]{4}{*}{$\mathrm{Au}$} & $\mathrm{O}(2 \mathrm{c})$ & -0.30 & 0.00 & +0.12 & 2.18 & 3.64 \\
\hline & & $\mathrm{O}(4 \mathrm{c})$ & -0.39 & 1.00 & +0.10 & 2.88 & 3.08 \\
\hline & & Ti(up) & -0.18 & 1.00 & +0.03 & 2.95 & 3.57 \\
\hline & & Ti(down) & -0.31 & 1.20 & +0.08 & 2.50 & 3.62 \\
\hline \multirow[t]{7}{*}{$\mathrm{TiO}_{2} / \mathrm{Pt}$} & \multirow[t]{2}{*}{ K } & $\mathrm{O}(4 \mathrm{c})$ & -3.22 & 0.00 & +0.91 & 2.80 & 3.40 \\
\hline & & Ti(up) & -3.42 & 1.00 & +0.91 & 2.79 & 3.37 \\
\hline & \multirow[t]{3}{*}{$\mathrm{Pt}$} & $\mathrm{O}(4 \mathrm{c})$ & -2.87 & 0.00 & -0.56 & 2.85 & 2.31 \\
\hline & & Ti(up) & -3.26 & 0.00 & +0.21 & 1.96 & 2.29 \\
\hline & & Ti(down) & -4.06 & 0.00 & +0.01 & 2.02 & 2.72 \\
\hline & \multirow[t]{2}{*}{$\mathrm{Au}$} & $\mathrm{O}(4 \mathrm{c})$ & -1.29 & 0.00 & -1.28 & 2.95 & 2.72 \\
\hline & & Ti(up) & -1.76 & 0.00 & -0.30 & 2.98 & 2.66 \\
\hline \multirow[t]{6}{*}{$\mathrm{TiO}_{2} / \mathrm{Ag}$} & $\mathrm{K}$ & $\mathrm{O}(4 \mathrm{c})$ & -2.86 & 0.79 & +0.91 & 2.85 & 3.46 \\
\hline & \multirow[t]{2}{*}{$\mathrm{Pt}$} & Ti(up) & -1.87 & 2.32 & +0.27 & 2.07 & 2.41 \\
\hline & & Ti(down) & -3.64 & 2.51 & +0.03 & 2.04 & 2.70 \\
\hline & \multirow[t]{3}{*}{$\mathrm{Au}$} & $\mathrm{O}(2 \mathrm{c})$ & -0.45 & 1.22 & +0.16 & 2.18 & 3.67 \\
\hline & & $\mathrm{O}(4 \mathrm{c})$ & -1.31 & 1.48 & -0.26 & 2.89 & 2.72 \\
\hline & & Ti(down) & -0.45 & 3.40 & +0.13 & 2.22 & 3.60 \\
\hline
\end{tabular}


Table S9. Adsorption of $\mathrm{K}$, $\mathrm{Pt}$ and $\mathrm{Au}$ single atoms on free-standing $\mathrm{ZrO}_{2}, \mathrm{ZrO}_{2} / \mathrm{Pt}(111)$ and $\mathrm{ZrO}_{2} / \mathrm{ZrPt}(111)$. Adsorption energy $(\mathrm{De}, \mathrm{eV})$, total magnetization $(\mathrm{M},|\mathrm{e}|)$, Bader charge of the adsorbed atom $\left(\mathrm{q}_{\mathrm{A}},|\mathrm{e}|\right)$, adatomoxygen minimum distance $\left(\mathrm{R}_{\mathrm{O}}, \AA\right)$ and adatom-Zr minimum distance $\left(\mathrm{R}_{\mathrm{Zr}}, \AA\right)$.

\begin{tabular}{|c|c|c|c|c|c|c|c|}
\hline Support & Atom & Site & De $(e V)$ & $\mathrm{M}(|\mathrm{e}|)$ & $\mathrm{q}_{\mathrm{A}}(|\mathrm{e}|)$ & $\mathrm{R}_{\mathrm{O}}(\AA)$ & $\mathrm{R}_{\mathrm{Zr}}(\AA)$ \\
\hline \multirow[t]{6}{*}{$\mathrm{ZrO}_{2}$-free } & \multirow[t]{2}{*}{$\mathrm{K}$} & O(down) & -0.91 & 0.00 & +0.90 & 2.69 & 3.71 \\
\hline & & $\mathrm{Zr}$ & -1.04 & 0.01 & +0.89 & 2.80 & 3.28 \\
\hline & \multirow[t]{2}{*}{$\mathrm{Pt}$} & O(up) & -2.04 & 0.07 & -0.08 & 1.95 & 2.91 \\
\hline & & $\mathrm{Zr}$ & -1.19 & 0.09 & +0.09 & 2.51 & 2.50 \\
\hline & \multirow[t]{2}{*}{$\mathrm{Au}$} & O(up) & -0.42 & 0.42 & -0.08 & 1.99 & 3.13 \\
\hline & & $\mathrm{Zr}$ & -0.44 & 0.41 & -0.01 & 2.94 & 2.95 \\
\hline \multirow[t]{7}{*}{$\mathrm{ZrO}_{2} / \mathrm{Pt}$} & \multirow[t]{2}{*}{ K } & O(up) & -2.73 & 0.00 & +0.88 & 2.60 & 3.47 \\
\hline & & $\mathrm{Zr}$ & -2.72 & 0.10 & +0.88 & 2.64 & 3.54 \\
\hline & \multirow[t]{3}{*}{$\mathrm{Pt}$} & $\mathrm{O}($ up) & -4.41 & 0.00 & -0.09 & 2.06 & 2.68 \\
\hline & & $\mathrm{O}($ down $)$ & -2.98 & 0.00 & -0.18 & 1.94 & 2.67 \\
\hline & & $\mathrm{Zr}$ & -2.73 & 0.00 & -0.14 & 1.96 & 2.63 \\
\hline & \multirow[t]{2}{*}{$\mathrm{Au}$} & O(up) & -1.78 & 0.00 & -0.58 & 2.86 & 2.94 \\
\hline & & $\mathrm{Zr}$ & -1.17 & 0.00 & -0.51 & 3.16 & 2.68 \\
\hline \multirow[t]{8}{*}{$\mathrm{ZrO}_{2} / \mathrm{ZrPt}$} & \multirow[t]{2}{*}{ K } & $\mathrm{O}($ down$)$ & -2.68 & 0.00 & +0.89 & 2.64 & 3.70 \\
\hline & & $\mathrm{Zr}$ & -2.53 & 0.00 & +0.90 & 2.71 & 3.43 \\
\hline & \multirow[t]{3}{*}{$\mathrm{Pt}$} & $\mathrm{O}($ up) & -2.20 & 0.00 & -0.08 & 1.96 & 2.65 \\
\hline & & $\mathrm{O}($ down$)$ & -3.47 & 0.00 & -0.09 & 2.04 & 2.81 \\
\hline & & $\mathrm{Zr}$ & -1.45 & 1.20 & -0.31 & 2.56 & 2.96 \\
\hline & \multirow[t]{3}{*}{$\mathrm{Au}$} & O(up) & -0.96 & 0.00 & -0.50 & 3.08 & 2.70 \\
\hline & & $\mathrm{O}($ down $)$ & -1.02 & 0.00 & -0.57 & 2.89 & 3.06 \\
\hline & & $\mathrm{Zr}$ & -0.95 & 0.00 & -0.50 & 3.09 & 2.70 \\
\hline
\end{tabular}

